\title{
¿El cinturón ecológico? Análisis de una marca urbana que nunca existió
}

Marie-Noëlle Carré. Centre de Recherches et de Documentation des Amériques (CREDA) - Université Sorbonne-Nouvelle - Paris 3, París, Francia.

Leonardo Fernández. Universidad Nacional de General Sarmiento, Buenos Aires, Argentina.

RESUMEN | ¿Cómo un proyecto territorial no concretado en su totalidad puede inscribirse dentro de la memoria metropolitana? En Buenos Aires, el cinturón ecológico, un dispositivo que se proponía ampliar la dotación de áreas verdes y limitar el crecimiento urbano, representa un caso emblemático. Con un análisis de las estrategias del marketing urbano desarrolladas para promocionarlo durante la última dictadura militar (1976-1983), este trabajo desentramará cómo este emprendimiento metropolitano, aunque nunca terminado, se transformó en una marca para Buenos Aires. Abordará su difusión mediática primero dentro de la puesta en escena de una crisis urbana; luego en la constitución de un nuevo proyecto de ciudad y, finalmente, en la promoción de un equipamiento de calidad a escala regional. A modo de cierre, considerará la inversión de esta imagen, a la luz de los conflictos metropolitanos actuales.

PALABRAS CLAVE | planificación territorial, áreas metropolitanas, medioambiente urbano.

ABSTRACT | How can an uncompleted territorial project brand itself in a metropolitan memory? In Buenos Aires (Argentina), the Cinturón ecológico (Green Belt) can be seen as a paradigmatic case to answer this question: even if the initial green ring around the city was partially implemented, its image seems to have had such a strong impact in the society that it still is a landmark in the metropolitan management discussions. This article explains how the urban marketing strategies displayed during the last dictatorship (1976-1983) participated to claim the Cinturón ecológico as an urban brand. It discusses how the 70 s urban crisis was directed in order to justify that infrastructure as a new urban project. Then, it shows how it was promoted as an efficient undertaking at the regional scale. Finally it questions how the image has gone through a reversal, confronted with current environmental conflicts.

KEY WORDS | territorial planning, metropolitan areas, urban environment. 


\section{Introducción ${ }^{1}$}

Con la conferencia de Estocolmo de 1972 se actualizan en numerosas urbes del mundo debates sobre el crecimiento urbano, con un nuevo impulso en torno al concepto de 'medioambiente'. ¿Cómo contener la expansión de las ciudades y mejorar la calidad de vida de sus habitantes sin atentar contra el medioambiente? En Buenos Aires, este contexto internacional tuvo un particular impacto durante el régimen de facto (1976-1983). Resurgieron preguntas sobre la urbanización que ya se habían planteado varias décadas antes, en un clima de reflexión que favoreció el surgimiento de experiencias novedosas en ruptura con el urbanismo clásico, que intentaban dar solución a problemas identificados como sistémicos. Entre ellos, el cinturón ecológico representó una voluntad de combinar, al mismo tiempo, un problema de espacio y de regulación para la disposición de los residuos, un límite físico a la ciudad, y la falta de espacios verdes. Sin embargo, el programa original de un anillo verde, previsto como regulador de la expansión metropolitana, nunca fue completado y hoy se reduce a tres sitios de disposición final de los residuos y a un tramo de autopista de 17 kilómetros en el noroeste de la región urbana. Este fracaso parcial se agrega a los más de cuatrocientos planes urbanos no logrados que experimentaron las ciudades argentinas entre 1940 y 1990 (Torres, en Dubois-Maury, 1990, p. 702). Sin embargo, la expresión "cinturón ecológico" sigue siendo empleada en la prensa o en grupos de decisión y de protesta para referirse a los equipamientos de tratamiento de residuos o a la empresa coordinadora de sus actividades. ¿Cómo un objeto que nunca correspondió al proyecto programático inicial llegó a imponerse con fuerza en la memoria territorial urbana?

La mayoría de los trabajos sobre la cuestión identifican el cinturón ecológico como un dispositivo socio-técnico-territorial enmarcado en las estrategias autoritarias de reconfiguración urbana de Buenos Aires. Pocos trabajos, en cambio, se dedicaron a cuestionar la articulación entre la infraestructura construida y su imagen proyectada. La operación, en su inicio, se benefició de una fuerte visibilidad mediática y alimentó claramente una retórica del ordenamiento y de la modernización urbana en un momento en que la puesta en escena de la reestructuración urbana (Greenberg, 2011) constituía una estrategia nacional en Argentina para asentar el posicionamiento de Buenos Aires entre las urbes más dinámicas y modernas del mundo. Hoy en día, es este producto contradictorio, complejo, entramado, el que parece cristalizarse con valor de modelo o de antimodelo en los debates metropolitanos sobre el ambiente o sobre la planificación urbana en el Gran Buenos Aires. Por lo tanto, en este trabajo apuntamos a cuestionar cómo se posiciona el cinturón ecológico en un proceso más amplio de marcamiento urbano (urban branding) de Buenos Aires. Destacaremos primero cómo la puesta en escena del cambio urbano en Buenos Aires y para sus habitantes se inscribe en contrapunto a otra puesta en escena mediática: la crisis urbana. Después, mostraremos cómo la marca en el es-

1 Este artículo debe mucho a la Ing. A. Álvarez, bibliotecaria del centro de documentación de la Coordinación Ecológica Área Metropolitana Sociedad del Estado (CEAMSE). La agradecemos por su ayuda archivística. 
pacio urbano, fuertemente mediatizada, se inscribe como proyecto de ciudad en Buenos Aires. Para terminar, analizaremos cómo la ingeniería sanitaria sirve para promocionar a nivel latinoamericano el uso de una infraestructura de vanguardia.

\section{La puesta en escena de tres crisis metropolitanas}

Cuadras sin asfaltar, recaudación deficiente, presupuesto municipal devorado por los sueldos municipales, hospitales degradados: al leer el diario Clarín del 23 de abril de 1976, el relato del discurso de ascenso a la intendencia del gobierno de facto de la Municipalidad de Buenos Aires, pronunciado por el brigadier Cacciatore, parece remitir a una ciudad en estado de abandono. Este artículo de prensa marca el comienzo de una larga y sostenida mediatización de los inicios, de los avances y las inauguraciones de las obras de infraestructura llevadas a cabo desde el Ministerio de Obras Públicas, que construyen la imagen de una ciudad en crisis. Ese punto de partida de la retórica gubernamental abarca un conjunto de problemáticas en la gestión urbana que, después de haber sido cuestionadas académica o técnicamente en las décadas previas, se plantean nuevamente durante los años 1970 en la esfera mediática. En este contexto, la mediatización de una ciudad sucia, congestionada, contaminada, concentrada puede analizarse como "una puesta en escena destinada sobre todo a los habitantes, para que acepten el proyecto de [una nueva] ciudad" (Rosemberg-Lasorne, 1997), destinada a "quien la merece" (Del Cioppo, 1980).

En el periodo 1976-1978 recrudece en los medios de comunicación la publicación de artículos y de archivos especiales vinculados con el urbanismo, la ecología y la calidad de vida en la ciudad. Específicamente, se concentran en tres cuestiones relacionadas con obras de infraestructura - como lo es el cinturón ecológico-implementadas por la Secretaría de Obras Públicas: la congestión urbana, la falta de espacios verdes y la contaminación atmosférica. Tales problemáticas aparecen por separado en los medios de comunicación de esos años, como lo muestran algunos títulos de artículos de diario: "La batalla por los espacios verdes" (La Nación, 26 de septiembre de 1976, pp. 12-16), "Autopistas: con veinte años de demora" (Clarín, 6 de mayo de 1977, p. 14), "La muerte de incinerador" (Clarín, 14 de enero de 1977). Se arraigan en una reflexión desarrollada a partir de los años treinta y cuarenta, que inicialmente apuntaba a una mirada integral de los problemas urbanos. Los trabajos de Carlos M. Della Paolera constituyen un punto de partida fundamental para analizar esta transformación. Heredero de la corriente de urbanismo organicista, primer titular de la cátedra de Urbanismo de la Universidad de Buenos Aires (1932) y jefe del primer plan regulador de Buenos Aires (1932), fue uno de los primeros en difundir bases para la planificación urbana, con influencia en los urbanistas, geógrafos, arquitectos e ingenieros civiles que accedieron a los cargos técnicos de la ciudad en 1976. Sus reflexiones, difundidas en numerosos artículos de diarios a partir de 1926, se anclan en la observación del crecimiento exponencial de Buenos Aires a partir del comienzo del siglo XX. Frente a una urbanización descontrolada, precaria (Liernur, 1993) e insalubre de la ciudad desde el fin del 
siglo XIX, el urbanista se focaliza, entre otras cosas, sobre la "desnaturalización del medio ambiente en la ciudad moderna" (Della Paolera, en Randle, 1977, p. 15), una temática que preocupa también a los ingenieros sanitarios en la misma época (Paiva, 1999). Della Paolera, siempre en Randle (1975), señala que en vez de ser un cuerpo sano y equilibrado, donde los habitantes gozan del acceso a "aire, sol y vegetación” (p. 55), el Gran Buenos Aires corre el riesgo irreversible de enfrentarse con todos los males de la urbanización desordenada: "contaminación sin alternativas, carencias de válvulas de escape a las tensiones de la vida urbana, juventudes malformadas" (p. 16). Destaca la inadecuada urbanización de la ciudad frente al nuevo modo de transporte rápido que es el automóvil. Propone un ordenamiento del tránsito de los peatones, de los automóviles y de la red subterránea de ferrocarriles primero en la ciudad de Buenos Aires, la más poblada, y luego en un plano regional, para planificar el futuro urbano de la metrópoli (pp. 97-106) y remediar tanto la contaminación atmosférica como la congestión urbana que amenaza a diario. Finalmente, Della Paolera insiste en que la urbe padece una fuerte división político-administrativa que la perjudica en su posicionamiento respecto a las otras ciudades del mundo, y alienta la creación de un Gran Buenos Aires (pp. 118-119).

En los años setenta, estas mismas discusiones se dieron nuevamente a raíz de las conferencias sobre el medioambiente en Estocolmo (1972), sobre población en Bucarest (1974), alimentos en Roma (1974), establecimientos humanos en Vancouver (1976), agua en Mar del Plata (1977). Sin embargo, aunque en Buenos Aires se daba un crecimiento urbano incesante y un cambio radical del modelo de transporte, sus problemas eran mucho menores que los encontrados por ciudades donde la transición demográfica y las migraciones desde los espacios rurales habían incrementado la presión urbana, como Caracas o México. Las discusiones se desarrollaban alrededor de un enfoque sistémico sobre el medioambiente y la salud, con fuerte resonancia internacional, y que aparecía solo en filigrana en los trabajos de Della Paolera. La población se multiplicó por dos desde 1940 a mediados de los setenta, y la cantidad de automóviles lo hizo por diez entre 1945 y 1975, pasando a 1,5 millones de unidades (Municipalidad de Buenos Aires, 1976, p. 14). Los edificios de departamentos estaban equipados de incineradores para quemar la basura domiciliaria. Esta medida, destinada a limitar la saturación de la quema municipal en la Capital Federal, originó una fuerte contaminación atmosférica descontrolada y amenazante para la seguridad sanitaria (Ríspoli, Leva, Crivelari \& Comerci, 1962). El tercer y el cuarto seminarios técnicos sobre la contaminación urbana, llevados a cabo en 1972 y 1974 y patrocinados por autoridades municipales, provinciales y nacionales, constituyen una buena fotografía de las inquietudes que conforman la cuestión ambiental. Reflejan también el dinamismo de la Ingeniería Ambiental argentina, que apuntaba a igualar la de países centrales y lideraba la profesión en América Latina. Los encuentros dieron cuenta de la diversidad de las temáticas abordadas — ruidos de tránsito, incineradores, contaminación, uso del suelo urbano- y del objetivo perseguido: "Promover el intercambio de la información técnica que concurra a 
la adopción de medidas concretas, conducentes a impedir el deterioro del medio urbano con miras a la preservación de la salud" (Tercer Seminario sobre la contaminación urbana, 1972). Como en los años treinta, la regulación y la limitación de la expansión urbana aparecen como temáticas de primer orden inscritas en la agenda de la gestión metropolitana.

A partir de 1977, sobre todo en los primeros años del régimen de facto, esta actualidad científica y técnica se difundió ampliamente en los medios de comunicación. Se constata por esos años una cierta correspondencia entre los eventos científicos y la difusión de las problemáticas abordadas en los grandes diarios nacionales, difusión que se organiza en torno a tres ejes: la justificación de políticas públicas de ordenamiento de la ciudad; la concientización del ciudadano mediano respecto de las cuestiones ambientales urbanas; y la demostración de una política eficaz, eficiente y veloz hacia los habitantes de la ciudad y los observadores foráneos. Concordamos con Rosemberg-Lasorne (1997), que plantea el acto de comunicación como el nodo central de un proyecto de ciudad, el cual opera como anuncio de "intenciones que se convertirán en decisiones" y sirve para instalar "el sistema de actores que concibe el proyecto". Sin embargo, hay que preguntarse si — como lo plantea Oszlak (1991) dicho acto no desencadenó una verdadera ruptura política y urbanística. La estrategia de puesta en escena de la crisis urbana apuntó a identificar actores — víctimas y decisores - y a definir problemas que hasta ahí parecían aislados, pero cuya resolución cabía dentro de un único proyecto. El vecino promedio de Buenos Aires aparece como el que le puede "reclamar a su intendente" (Clarín, 1978, 14 de abril). Las temáticas abordadas, a veces en un tono dramático, a través de descripciones del ambiente urbano, de la marginalidad o de la ingobernabilidad, daban eco a las Cartas de Lectores difundidas por los diarios. Sin embargo, a pesar de la promoción de esta participación ciudadana, la definición de la planificación urbana se hizo en su gran mayoría sin que los habitantes de Buenos Aires pudieran opinar, en una dinámica de decisión desde arriba hacia abajo. Se puede generalizar esta observación a la mayoría de las operaciones de planificación urbana llevadas a cabo en aquella década, sea en América Latina o en países centrales. En Buenos Aires, esta modalidad tuvo un impacto decisivo. Sobre la opacidad de los procedimientos utilizados durante la dictadura se construyeron los movimientos ciudadanos de protesta contra los rellenos sanitarios a fines de los años 1990. Por otro lado, la puesta en escena de la restauración urbana se focalizó en el papel desempeñado por algunos funcionarios públicos: el intendente y los responsables de la Secretaría de Obras Públicas de la ciudad de Buenos Aires. Protagonizaban los anuncios de reformas urbanas, que aparecían como un contrapunto a las descripciones desastrosas de la ciudad en títulos con voz activa y predictiva: "Será combatida en la ciudad la contaminación ambiental" (Clarín, 1976, 31 de diciembre); "Contrataron la construcción de dos autopistas" (Clarín, 1978, 20 de enero). La fuerte fragmentación entre las distintas temáticas refleja cómo se había desplazado el abordaje de las cuestiones del ambiente urbano: si anteriormente estaban a cargo del urbanismo, y aunque este hubiera resurgido como disciplina en los debates sobre 
la metrópoli, era ahora la ingeniería la que se encontraba al centro de la resolución de las problemáticas urbanas.

La problematización de la crisis urbana y el énfasis puesto en la urgencia de medidas urbanísticas radicales, tanto en la ciudad como en la metrópoli, debe entenderse como una estrategia para posicionar Buenos Aires en el mundo como urbe de nivel internacional. Ocurrió en un momento crítico, en que la apuesta mayor para la Municipalidad de Buenos Aires era la recepción del Campeonato Mundial de Fútbol, en el año 1978. En ese entonces, la reafirmación del "rango capitalino a nivel nacional, y metropolitano a nivel mundial” (Consejo de Planificación Urbana, en Oszlak, 1991, p. 78) de la metrópoli permitió justificar la implementación y la difusión mediática del gran número de instrumentos de regulación y legislación editados en esos primeros años de intendencia. El Secretario de Obras Públicas de la Ciudad de Buenos Aires aprovechó el día mundial del urbanismo celebrado el 9 de noviembre de 1976, para elogiar las tareas de planificación desarrolladas en su tiempo por Della Paolera y para afirmar que Buenos Aires siempre estuvo "a la vanguardia de las grandes ciudades" (Clarín, 1977, 9 de noviembre). El artículo "Buenos Aires for export", publicado en el diario Clarín del 14 de mayo de 1978, refleja la especulación sobre la presentación a los turistas internacionales de una ciudad intervenida por el arquitecto-urbanista de renombre internacional Le Corbusier, "erizada de edificios", "dotada de instrumentos legales para seguir creciendo con reglas de sistematización", de "tránsito distendido por las autopistas", exenta de "contaminación ambiental". Entonces, se puede interpretar el "lapso verdaderamente insólito" en que se desarrollan obras tan monumentales como el cinturón ecológico, el Parque Interama, las autopistas 25 de Mayo y Perito Moreno como una medida estratégica que apunta a rehabilitar el esplendor de la ciudad y diluir todo rasgo de crisis urbana.

Greenberg, en su libro sobre Nueva York (2008), analiza cómo, en la misma época, un grupo de empresarios y de funcionarios desarrolló un fuerte marketing urbano destinado a cambiar la imagen de la ciudad en crisis, usando el proceso del marcado urbano - urban branding-, aunque este se desarrollara avant la lettre. En el caso de Buenos Aires, procesos semejantes parecen haberse desarrollado con la obra de las autopistas, los parques urbanos, los emprendimientos inmobiliarios y del cinturón ecológico. Este último, por apuntar a modificar tanto la circulación urbana como la estructura de los espacios verdes y también la recolección y el tratamiento de los residuos urbanos, merece un desarrollo particular dentro del análisis de la puesta en escena de la transformación y modernización de Buenos Aires.

\section{El cinturón ecológico: ¿una marca metropolitana?}

Dentro del abanico de obras de infraestructura llevadas a cabo entre 1976 y 1983 , el cinturón ecológico merece un lugar aparte por su carácter de "proyecto integral" (Laura, 1974, p. 55). Se constituye como una piedra angular para una ciudad ordenada y moderna. Encarna una marca urbana transformadora, un sello territorial 
sobre la metrópoli. Como lo indican Dumont y Devisme (2006), "el instrumento de comunicación territorial (...) es el componente de escenas que son ellas mismas focales del conjunto de un proyecto y constituyen a su vez el prisma de estrategias y de dinámicas territoriales metropolitanas". La puesta en escena del cinturón ecológico, a través de los elementos de su difusión, aparece, entonces, como reflejo de una imagen de la metrópoli caracterizada esencialmente por su centro y su borde.

La instalación del cinturón ecológico despertó el interés de investigadores, en una perspectiva histórica, respecto a la disposición de los residuos (Paiva, 2003; Suárez, 1998; Prignano, 1998). Este proyecto regional, presentado como una alternativa integral a la contaminación, la congestión, la concentración urbana, surgió a partir del libro programático y epónimo del abogado Guillermo D. Laura (1974), y fue propulsado a la realización cuando el intendente Cacciatore nombró a su ideólogo como Secretario de Obras Públicas de la Municipalidad de Buenos Aires, en 1976. La amplitud del proyecto, como objeto geográfico de escala regional, traza una clara continuidad con las obras infraestructurales llevadas a cabo en el periodo anterior, tanto en la Argentina como en otros países de la región y, más ampliamente, en el contexto desarrollista posterior a la Segunda Guerra Mundial. Las grandes represas (Salto Grande), la represa de Itaipú en la frontera brasileñoparaguaya, la construcción de Brasilia (Brasil) en el transcurso de los años 1960 y el comienzo de los años 1970 son representativas de estas iniciativas implementadas desde el nivel estatal federal. Planifica la realización de un medio-anillo de 150 kilómetros de largo sobre una extensión de 14.250 hectáreas, de río a río; una afectación exclusiva de estas tierras a espacios verdes de recreación o de agricultura urbana; la realización de cuatro sitios de disposición de residuos con la tecnología del relleno sanitario y una autopista circundante al área metropolitana, conectada con los otros proyectos de vías rápidas previstas por el gobierno (Figura 1). Como lo muestran los documentos, la dimensión gráfica del proyecto se concentra sobre la localización de este anillo verde urbano, cuya precisión de trazado varía según el objetivo del documento. Las representaciones libres y creativas del diario $\mathrm{La} \mathrm{Na}$ ción y de las Memorias y Balances de 1980 ilustran un esquema radial cuyo centro de gravedad es la Ciudad de Buenos Aires. Esta representación, que refiere a un clásico modelo centro-periferia, se inscribe en la política llevada a cabo por la Intendencia de Municipalidad de la Ciudad de Buenos Aires. Según algunos autores, se trata de hacer de la ciudad una zona relativamente exclusiva para un sector específico de la sociedad, una burguesía urbana (Ozlak, 1991). Promulgadas por la ciudad, desde la ciudad y para la ciudad, las políticas de ordenamiento y de planificación urbana se caracterizan por este esquema que marca el centro y la periferia, y que hace del conurbano bonaerense - el espacio de la industria, de los obreros, muy poblado y pobre- un espacio en blanco. La mayoría de las veces está sencillamente borrado del mapa, o salpicado de escasas viviendas campestres con una imagen muy distante de la situación de aquel entonces (Figura 2). La Capital Federal "erguida de edificios", delineada y colorida, y su complemento, el cinturón ecológico, recorrido por las automóviles y trazado por árboles, simbolizan la ciudad moderna y desarrollada. 
FIGURA 1 | El cinturón ecológico. Un proyecto metropolitano para la capital argentina

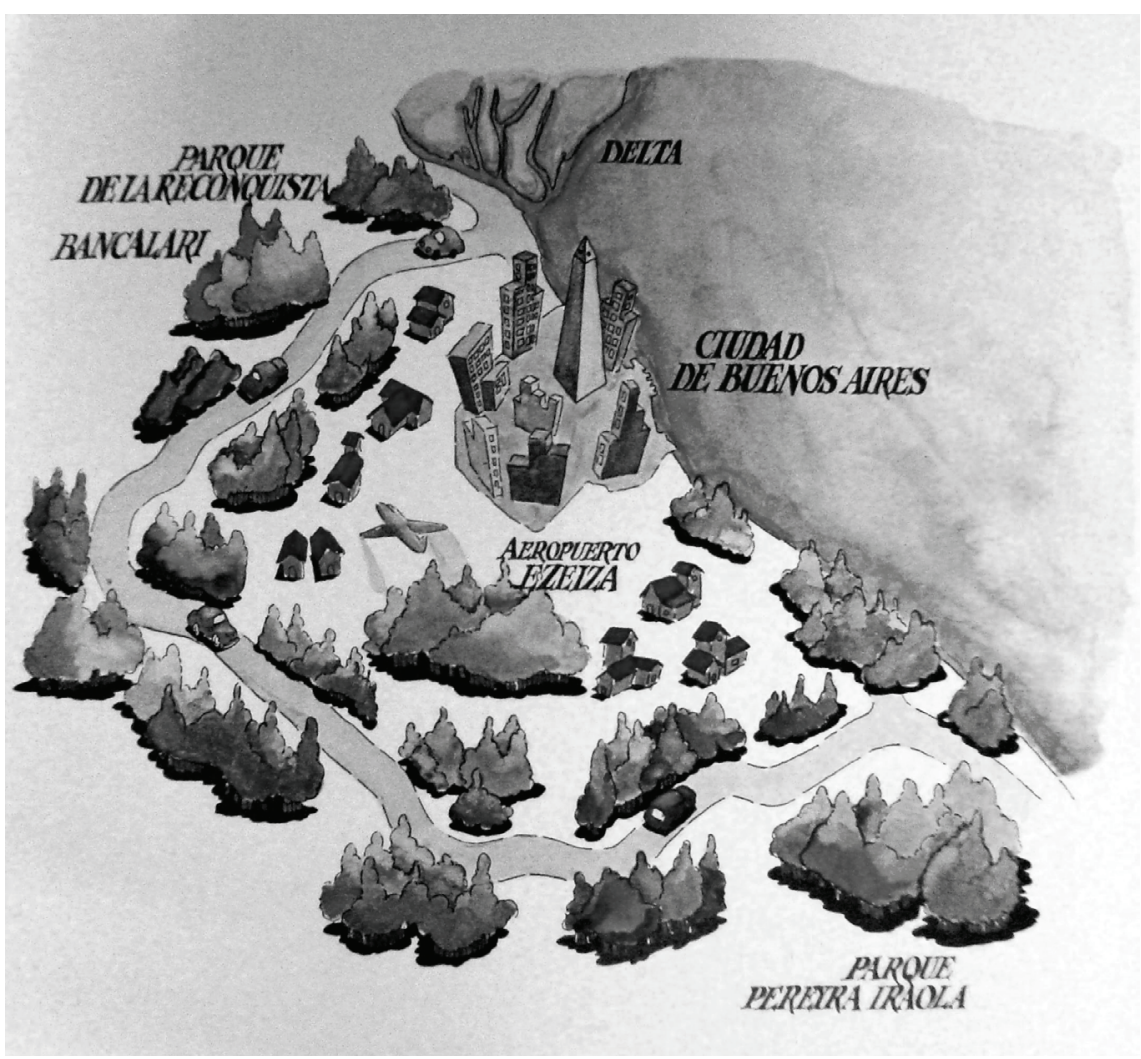

FUENTES LAURA, 1979; ATLAS AMBIENTAL DE BUENOS AIRES (www.ATLASDEBUENOSAIRES.edU.AR); ENTREVISTA CON A. GARAY, EX SUBSECRETARIO DE URBANISMO Y ViVIENDA DE LA PROVINCIA DE BUENOS Aires, 7 DE MAYO DE

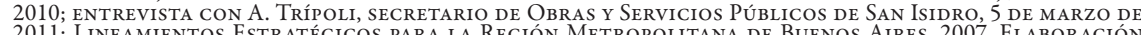
2011; Lineamientos Estratégicos para la Región Metropolitana de Buenos Aires, 2007. Elaboración PROPIA, 2011.

Pero además de este proyecto socioeconómico y político, la creación de un cinturón ecológico entre la ciudad y el interior del país se ancla en otra referencia anterior explícita, mencionada por el mismo G. Laura. Preexisten proyectos de cinturones verdes en grandes urbes contemporáneas al proyecto - el Green Belt de P. Abercrombie en Londres (1944), el cinturón verde de Seúl construido en 1971 bajo el régimen de Park Chung-Hee-. Sin embargo, para el cinturón ecológico, Laura toma como modelo la construcción del parque Tres de Febrero — también conocido como Bosques de Palermo- sobre terrenos bajos que fueron rellenados con residuos bajo el gobierno progresista de D. F. Sarmiento. Esta operación, llevada a cabo a finales del siglo XIX, se articulaba con el proyecto más amplio de civilizar progresivamente un espacio nacional teorizado como "bárbaro". La moviliza- 
ción de la grilla y del parque (Gorelik, 1998), o sea, de la trama urbana regular y de la naturaleza domesticada como herramientas del proyecto modernista, reaparece como herramienta del ordenamiento urbano a escala metropolitana en el proyecto de cinturón ecológico. En este sentido, las representaciones del cinturón ecológico en los medios de comunicación concentran numerosas referencias urbanísticas y culturales foráneas, o históricas, que se plasman en la puesta en escena de la marca metropolitana. El cinturón ecológico está presentado primero como una obra destinada a crear espacios verdes y a activar la circulación en automóvil. Como lo veremos más adelante, la disposición de los residuos aparece o como una cuestión técnica cuya difusión se circunscribe a ámbitos determinados, o como una operación a corto plazo preliminar a la implementación de los dos otros objetivos. De hecho, en los estatutos de la Coordinación Ecológica Área Metropolitana Sociedad del Estado (CEAMSE), la ocupación principal de la empresa del Estado está definida, hasta 1984, como de "recuperación de tierras bajas y parquización"; y el mismo G. Laura especifica que "el objetivo central del proyecto no era la basura sino hacer parques". Esta puesta en escena de los dos aspectos más atractivos de la marca urbana que constituye el cinturón ecológico pasa por abundantes representaciones de lo verde y de la obra de infraestructura vial en las Memorias y Balances de la empresa y en los boletines Noticias CEAMSE. En estos dos últimos tipos de documentos, así como en ciertas ponencias presentadas en congresos (Bellucci, Gil \& García, 1982), el "parque recreativo" es la expresión que predomina sobre la de relleno: parque recreativo de La Reconquista, parque recreativo Bancalari... Este enfoque específico, que remite al porvenir - en un mediano plazo - el desarrollo de los sitios de disposición de los residuos, se articula con una copiosa presencia mediática (Clarín, 8 de enero de 1977, p. 12; Clarín, 21 de abril de 1977, p. 21; Clarín, 7 de mayo de 1877, p. 16; La Nación, 7 de mayo de 1977, p. 20; Clarín, 4 de junio de 1977, p. 6) de la recuperación de áreas verdes metropolitanas. Laura indica, además, que la propuesta de creación de parques metropolitanos dirigida a los municipios constituyó una estrategia discursiva para que estos manifestaran un acuerdo de principio respecto al proyecto (Laura, entrevista personal, 24 de junio de 2010).

Limitarse a esta observación sugeriría que los objetivos del cinturón ecológico no fueron vistos de la misma forma por los ciudadanos, los empresarios o las instituciones en los medios de comunicación. Ciertamente, la obra se implementó en un marco autoritario donde el debate sobre decisiones ejecutivas estaba reducido a su mínima expresión. Sin embargo, la marca física del cinturón ecológico y su puesta en escena cristalizaron otras informaciones "sobre las representaciones integradas al proyecto, (...) las racionalidades de los actores (Lussault, 1996), sus mundos de valores y de mitologías (Rosemberg-Lasorne, 2000)”. Ello puesto que la jerarquización de los objetivos del cinturón ecológico se articuló también estrechamente con las proyecciones que el primer equipo ejecutivo plasmó en la marca física del cinturón ecológico.

Estas diferencias, más que divergencias, dejan a la vista distintos grados de teorización del proyecto, desde un acercamiento pragmático hasta una dimensión visio- 
naria. Sobre todo, muestran el cinturón ecológico como marca urbana que cristaliza la circulación de modelos internacionales de acción conjunta sobre la ciudad y su ambiente. Laura, en su libro El cinturón ecológico, inscribe la obra en la trayectoria de experiencias exitosas de Estados Unidos, como el aeropuerto La Guardia, y la recuperación de la ciudad de Berlín desde sus escombros, después de la Segunda Guerra Mundial, en Alemania. Si bien estas infraestructuras sirven de garantía técnica y social, la innovación de Laura consiste en proponer la planificación de una obra-suma que integraría tanto la gestión de los residuos como la organización del tránsito y la preservación de espacios verdes en una infraestructura sistémica a escala regional. La dimensión pragmática del proyecto — dar soluciones prácticas a problemas puntuales en un momento idóneo- se inscribe explícitamente en una línea política progresista y liberal, que refleja la admiración del abogado-planificador por el modelo liberal planteado por los Estados Unidos. A modo de eco con esta obra, la referencia que hacen casi todos los miembros del equipo al informe sobre el Gran París titulado Réponse à la violence, de Peyrefitte, Schmelck y Dumoulin (1977), hace resonar con mayor cercanía la dicotomía enunciada por Sarmiento en su ensayo Civilización y barbarie: la marca física del cinturón ecológico actúa como zona de transición, de limitación y de separación entre la ciudad: la civilización; y el exterior (pampa, regiones lejanas, países limítrofes): la barbarie. Si bien esta temática controversial no aparece explícitamente, se formula la necesidad de recurrir a una "muralla verde" para controlar la expansión urbana sin límite (Bellucci), encauzar a los jóvenes a través del deporte (G. Laura), "restablecer el equilibrio a fin de reducir la agresividad de la vida de la ciudad” (Della Paolera). Por fin, Cl. Della Paolera, hijo del urbanista C. M. Della Paolera, director ejecutivo del CEAMSE entre 1977 y 1982, retoma el programa de Laura elevando la discusión al plano teórico al plantear el cinturón ecológico como un modelo de "metabolismo urbano". Esta dimensión visionaria abarca, por supuesto, mucho más que la sola obra de infraestructura. Es el sistema urbano completo que está cuestionado a través del prisma del cinturón ecológico, en el marco de una reflexión que se desarrolla a nivel internacional en esta misma época. En este contexto en que el cálculo de las entradas y las salidas urbanas de materia y de energía deviene un indicador para la ecología urbana, la presentación del metabolismo urbano del cinturón ecológico en Nueva York ante la Sociedad de Ingenieros Civiles norteamericanos adquiere importancia para la difusión del emprendimiento. El cinturón ecológico, más allá de una marca física, deviene la firma de Buenos Aires, una garantía de la calidad de vida en la metrópoli argentina.

Estas dos dimensiones del cinturón ecológico como proyecto de ciudad - la marca física en la metrópoli y la garantía de la calidad de vida urbana- se articulan a su vez con una promoción de la calidad tecnológica del emprendimiento metropolitano a escala internacional. Más allá de la dimensión metropolitana del emprendimiento, se proclama el uso de nuevos métodos de disposición de los residuos y la implementación de un organismo que, experimentando modalidades de privatización de servicio de avanzada, van cimentando el papel del cinturón ecológico como prototipo de una nueva gestión de los residuos a escala de las Américas. 
FIGURA 2 | El cinturón ecológico de Buenos Aires - Un "green belt" para una ciudad moderna

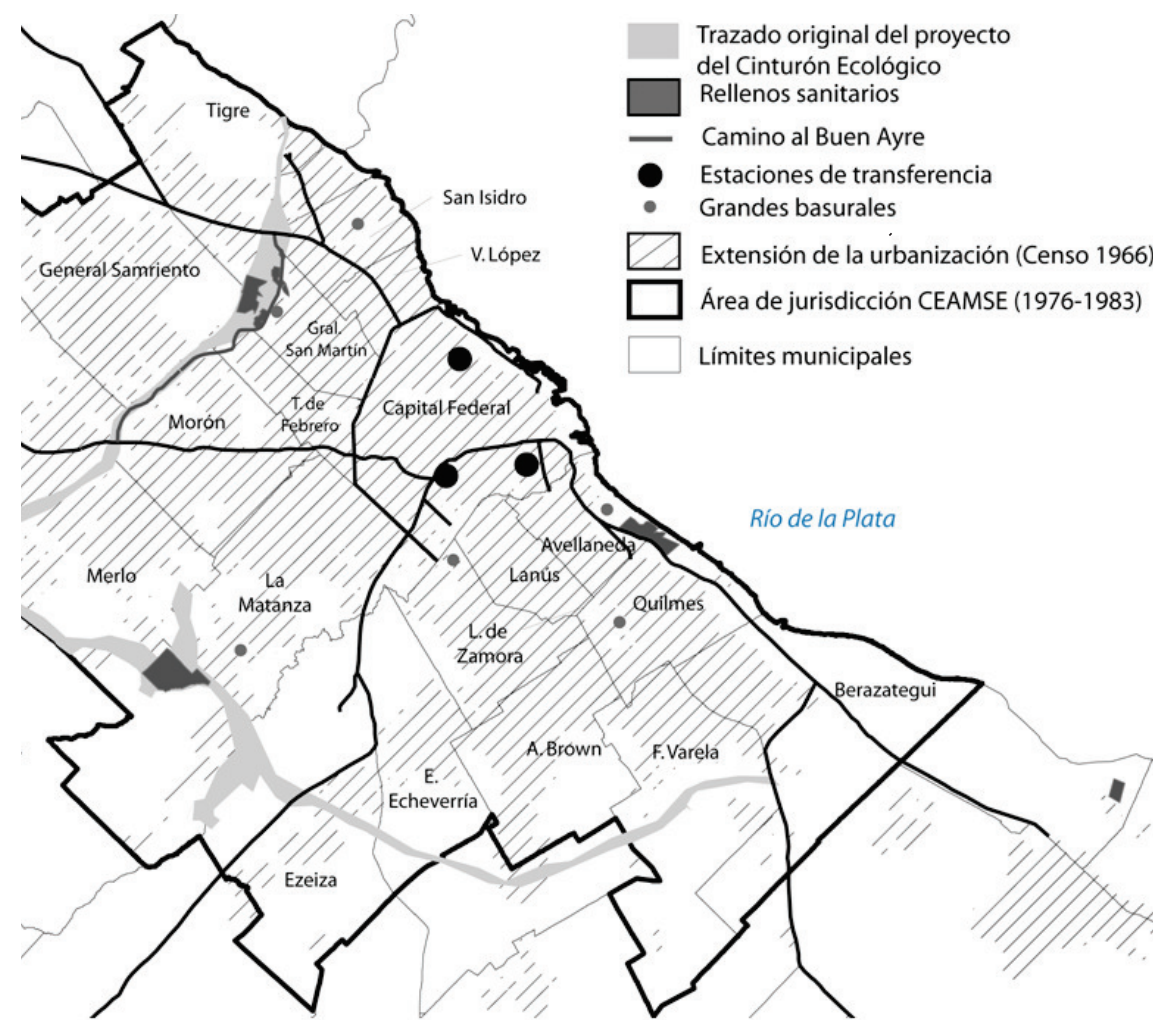

fuente Coordinación Ecológica Área Metropolitana Sociedad del Estado (CEAMSE), Memorias y BaLANCES, $1980(\mathrm{~s} / \mathrm{P})$.

\section{¿Un dispositivo experimental en América Latina?}

El relleno sanitario constituye un componente central del cinturón ecológico. Este dispositivo, formalizado por primera vez en Fresno (Estados Unidos, California), en 1937 (Melosi, 2004), consiste en acumular los residuos en celdas aisladas del suelo, de la atmósfera, de las aguas. Después de desarrollarse en ciudades europeas y mejorar considerablemente el impacto de la acumulación de los residuos sobre el ambiente urbano, se implementa gradualmente en América Latina durante los años setenta, primero en Chile y luego en Brasil y México. En Buenos Aires, la disposición de los residuos en los cuatro sitios que equipan el cinturón ecológico a partir de 1977 se define por su carácter sistemático. A partir de esta fecha, la Ciudad de Buenos Aires 
y los diecinueve municipios que conformaban en aquel entonces el Gran Buenos Aires por mandato legislativo, tienen que recolectar y trasladar los residuos generados en su jurisdicción a los rellenos sanitarios. El primer sitio es el de Bancalari, al noroeste del área metropolitana, a poca distancia de la ruta panamericana. El relleno de Villa Domínico se abre pocos meses después. Sin embargo, la difusión mediática de los rellenos sanitarios comienza antes de su construcción, y bajo los argumentos de la erradicación de los basurales a cielo abierto del sur de la Ciudad de Buenos Aires. Avanza sobre dos dimensiones de la técnica, que intervienen como contrapunto a las quejas de vecinos. Por una parte están las descripciones ("Máquinas de avanzado diseño bajo el control de expertos ingenieros", La Nación 25 de agosto de 1977, p. 14), que alternan con fotos o dibujos de sitios en obras, con caminos de tierra, celdas definidas, que completan este dispositivo tecnológico. Por otra, esta mediatización de "los beneficios del relleno sanitario" (Clarín, 12 de abril de 1978, p. 20) para eliminar la basura se completa con una información muy regular sobre la actualidad tecnológica en términos de tratamiento y acondicionamiento de los residuos.

$\mathrm{Al}$ mismo tiempo que se anunciaba la exclusividad del relleno sanitario como única salida económica viable para el tratamiento de los residuos en la Argentina (Clarín, 14 de enero de 1977, p. 12), noticias puntuales informaban sobre las investigaciones o innovaciones en curso en otros países: "Inventaron en Alemania petróleo artificial a partir de la basura"; "Nueva Era - combustible de basura" (Clarín, 13 de mayo de 1977, p. 26). También se presentó un intento de uso de los residuos como combustible alternativo al petróleo en una pequeña ciudad de Estados Unidos, en el contexto de la crisis energética internacional. La propaganda para el relleno sanitario difundía un método que, si bien aparecía como innovador, ya estaba superado por otras tecnologías en el mundo. Además, ya había sido puesto a prueba en la misma metrópoli de Buenos Aires, como dan cuenta los relatos de ingenieros sanitarios en Villa Domínico. El relleno sanitario apareció en la Argentina en el mismo momento en que en los países donde había sido experimentado se empezaba a dar paso a otras soluciones para los residuos. Por ejemplo, en Francia, la primera ley-marco sobre el reciclaje de los residuos se dictó en 1975; en Finlandia se promulgó en 1979 y en Dinamarca esta directiva apareció en 1981. ¿Por qué, a pesar de buscar y difundir la excelencia tecnológica, los decisores eligieron concretar, para la disposición de los residuos, una tecnología ya cuestionada en aquel entonces en términos de impacto ambiental?

\section{El relleno sanitario en los años 1970: ęuna innovación en América Latina o una dependencia técnica de países centrales?}

Esta cuestión se puede analizar bajo el ángulo del "path dependency" (David, 1985), una temática muy importante para la América Latina de los años setenta, sobre todo en su relación con Estados Unidos. A nivel de las Américas, la implementación del cinturón ecológico y de los rellenos sanitarios coincidió con un momento de discusión técnica sobre los métodos que permitirían mejorar el tratamiento de los residuos en las grandes ciudades de América Latina. Estos aglomerados urbanos 
estaban en pleno desarrollo demográfico y experimentaban un fuerte incremento de la producción de residuos, paralelo al aumento de la población. La Organización Panamericana para la Salud (OPS) fue uno de los primeros organismos en señalar el peligro sanitario que representaba una mala gestión o una ausencia de planificación del destino de los residuos. En agosto de 1974, la entidad organizó el primer simposio regional sobre Ambiente, Salud y Desarrollo, con un especial enfoque en las apuestas de la gestión de los residuos sólidos urbanos. Las conclusiones de este simposio cierran para América Latina el amplio panorama de alternativas que se abrían en ese momento a escala internacional. Promueven el relleno sanitario que "parece a todas luces para los países de Latinoamérica y del Caribe, la técnica más económica" (Alegre, 1978). Si bien los técnicos y expertos que se expresaban en el marco de eventos sobre el saneamiento en Argentina o en América Latina conocían y daban cuenta de los avances tecnológicos presentados previamente, así como de la necesidad de pensar en la separación en origen y la valorización de los residuos, la complejidad social y el alto costo económico que representaban esos nuevos métodos constituyeron un factor determinante para descartarlos en un contexto de urgencia sanitaria.

En aquel momento, si bien el proyecto del cinturón ecológico todavía no estaba en los planes, en la Argentina ya existían experiencias de racionalización, organización, conocimiento de sus residuos, como también sobre los métodos adecuados y las dificultades que se enfrentaban cuando se trataba de coordinar relevamientos y centralizar información. Por ejemplo, en 1969, el Ministerio Nacional de la Salud Pública desarrolló una encuesta nacional en las ciudades de más de 20.000 habitantes del país, menos la Región Metropolitana de Buenos Aires. La Facultad de Ingeniería Sanitaria de la Universidad de Buenos Aires realizó un estudio de calidad de los residuos de la Ciudad en 1972, y algunos municipios del conurbano efectuaron análisis puntuales, en la perspectiva de desarrollar métodos alternativos de tratamiento, como el compost (Alegre, 1978). Además, en los años 1980, la creación del Servicio Nacional de Basura (Senaba) apuntaba a que los núcleos urbanos de tamaño reducido (menos de 20.000 habitantes) pudieran recibir asesoramiento para mejorar la gestión de sus residuos. Esta capacitación incipiente les dio al país y a los técnicos especializados en la gestión de los residuos cierta autoridad en el tema, que se tradujo, por ejemplo, en el impulso de cursos de gestión de los residuos en el marco de la OPS. El valor de la experiencia se incrementó en el momento de la implementación de los rellenos sanitarios del cinturón ecológico, a partir de las normas dictadas por la American Society of Civil Engineers (ASCE) y la Agencia de Protección Ambiental de Estados Unidos. La aprobación del proyecto por el Banco Interamericano de Desarrollo (BID) y el compromiso financiero de la banca con el organismo que coordinaba el cinturón ecológico, el CEAMSE, le dio al emprendimiento una convalidación que lo ubicaba en el rango del modelo de la gestión deseable para las ciudades latinoamericanas. Este hecho constaba no sin orgullo en el editorial de las Memorias y Balances de 1978: 
Atendiendo a una invitación del Banco Interamericano de Desarrollo una misión del CEAMSE se trasladó a la Ciudad de Washington en el mes de octubre de 1978, para exponer el programa del Cinturón Ecológico ante las más altas autoridades del Banco. La misión integrada por dos miembros del Directorio de la Sociedad encontró una excelente disposición en el Banco para el tratamiento de problemas ecológicos y del medio ambiente, y en el curso de una audiencia privada con el presidente del Banco, se pudo confirmar el interés de la institución en participar activamente en un eventual financiamiento de nuestro programa. Como consecuencia de esta gestión la Misión Anual de Programación del Banco que visitó a la Argentina en el transcurso del presente mes de marzo, ratificó su interés de colaborar en la ejecución del programa del Cinturón Ecológico. (CEAMSE, Memorias y Balances, 1978)

Si bien están documentados los pasos de realización del relleno sanitario, resalta en Buenos Aires que la gran mayoría de los trabajos eluden la cuestión de la disposición de residuos en áreas inundables, criterio que fundamenta la creación del cinturón ecológico. De hecho, y en contra de un acercamiento sistémico a la ecología regional, los terrenos elegidos para la instalación de los rellenos sanitarios aparecen presentados en la mayoría de los trabajos como espacios sin valor: "Recuperación de áreas deterioradas” en el Primer Congreso de Ecología Urbana en Buenos Aires de 1982 (Bellucci, Gil \& García, 1982); o terrenos anegadizos. Por otra parte, el ordenamiento del basural de La Quema para su futura transformación en parque Almirante Brown, aparece difundido en los diarios como una primera implementación de la técnica del relleno sanitario. Sin embargo, el trabajo no consistiría en aislar la basura preexistente respecto del suelo, de la atmósfera y de las aguas del lecho mayor del Matanza-Riachuelo, sino en recubrirla de una capa de tierra para la parquización posterior.

\section{La ingeniería sanitaria, una profesión de la ciudad en las transformaciones urbanas}

Además de contribuir a plantear las bases de una gestión adecuada de los residuos, la promoción del cinturón ecológico sirvió para instalar en la escena central de los medios de comunicación una "profesión de la ciudad", la del ingeniero sanitario. Como lo plantea Paiva (1999), la ingeniería sanitaria se consolidaba lentamente en la Argentina a comienzos del siglo veinte (1890-1915), alrededor de una noción de ambiente urbano que nuclea los medios técnicos de abastecimiento de agua y de alcantarillado; los tipos de materiales que se deben usar según las características del medio; y los métodos de tratamiento de los residuos. En los años setenta, se observa un refuerzo de la imagen del ingeniero sanitario como actor de la cuestión ambiental, que conecta con la obra del cinturón ecológico a distintos niveles de análisis. Primero, y de forma general, los diarios relevaban el papel preponderante de este profesional urbano. En Clarin del 6 de junio de 1978, el titular del Centro Argentino de Ingenieros celebra el día de la Ingeniería Argentina efectuando un paralelo entre los periodos de mayor brillo del país y el despliegue de la actividad ingenieril. Destaca el papel desempeñado por estos profesionales en las obras de infraestruc- 
tura implementadas a partir de 1977. De la misma forma, el funeral del fundador de la firma Techint, Agustín Rocca, tiene una amplia cobertura mediática en los diarios del año 1978, con retrospectivas sobre la vida del ingeniero. Este "pionero de la siderurgia argentina", inmigrante italiano, emprendedor destacado, aparece entonces como una encarnación argentina del self-made man norteamericano. En este contexto, no sorprende tanto que el ensayo de Laura, El cinturón ecológico, haya sido galardonado con el premio Ing. Luis A. Huergo. Aunque su autor sea abogado, el proyecto que presenta se ubica en la misma línea que los principios de higienismo, los cuales sostuvieron la ingeniería sanitaria desde su creación. Por otra parte, el cinturón ecológico constituía una obra de infraestructura mayor en que se podrían desempeñar numerosos ingenieros civiles y sanitarios, en un mismo esfuerzo hacia la "anulación de la 'suciedad'" (Trelles, en Laura, 1979). Por fin, se observa que en las décadas de los sesenta y setenta, la parte de investigación de la Facultad de Ingeniería de la Universidad de Buenos Aires constituía un lugar donde se formaban los futuros miembros del personal técnico-administrativo del gobierno municipal o nacional, o de la misma CEAMSE entre 1977 y 1983, e incluso hoy. Este elemento tiene implicancias en la observación hecha por la Organización Panamericana de la Salud en el primer simposio sobre residuos sólidos, en 1974, respecto de la escasez de personal capacitado como ingeniero para la tarea de la gestión del ambiente. Pero también sirvió para propulsar en algunas empresas el desarrollo de la técnica de los rellenos sanitarios. De hecho, la presencia de la firma Techint, en pleno desarrollo en ese momento, representaba una marca de calidad y de excelencia para la operación del relleno de Villa Domínico, que se abría justo después de la experiencia de Bancalari, y para el transporte de residuos desde las estaciones de transferencia hasta los rellenos. De la misma manera, se consolidaba un esquema recurrente de asesoramiento técnico para la construcción de los rellenos, el control de estos y de la recolección, por parte de la firma norteamericana Wheeran Engineering Inc.

\section{El CEAMSE, una experimentación de la privatización periférica}

Además de equipamientos de disposición, la creación del CEAMSE se caracteriza por la aparición de una nueva forma de organización institucional. Greenberg (2008) comenta que en Nueva York se desarrolló un proceso similar, que apuntaba a incentivar el desarrollo económico con, por ejemplo, exenciones de impuestos para los nuevos emprendedores. En Buenos Aires, el cinturón ecológico significó una renovación institucional y económica que implicaba una privatización mayor de los servicios brindados tradicionalmente por el Estado. Esta dimensión es importante tanto para la transformación de Buenos Aires en ciudad moderna, como para la marca del cinturón ecológico. Se inscribe en un proceso desarrollado a escala nacional, y que Schvarzer (1981) identificó como "privatización periférica". En este caso, dicha expresión remite al proceso mediante el cual empresas del Estado centralizadas delegan la mayor parte de sus tareas a empresas privadas. Para los residuos, afecta tanto la recolección y la disposición como la construcción de las autopistas. La delegación del servicio de la recolección y de las obras de infraestructura del cinturón ecológico 
constituyó una estrategia de reforma a distintos niveles. Primero, sirvió para competir a nivel internacional, y sobre todo con Estados Unidos. Laura relata que la concesión del servicio de recolección en la ciudad de Buenos Aires se hizo mediante un pliego de licitación, lo que nunca había ocurrido antes, un proceso destacado tanto por el tamaño del contrato, "el (...) más grande de la historia, del mundo", como por los métodos empleados para la licitación. El abogado relata la transparencia y la velocidad del proceso, que se desarrolló en treinta días, en 1980. Hoy en día se cuestiona la primera de estas dos características, por los métodos propios del régimen de facto y los vínculos políticos y económicos estrechos que existían con círculos de decisión de Estados Unidos. Sin embargo, lo que se puede subrayar es que la presencia de intendentes de las "tres ciudades más grandes de Estados Unidos" y sus asesores dentro del comité técnico de adjudicación demuestra hasta qué punto igualarse a las ciudades norteamericanas constituía un objetivo para el gobierno urbano de facto de Buenos Aires. La participación de las tres empresas de recolección de residuos más grandes del mundo, entre las cuales estaba Waste Management Inc., ganadora de la licitación, significa también un criterio de competitividad relevante por el personal ejecutivo de la CEAMSE. Laura especifica que "E. S. Savas, el máximo asesor de Ronald Reagan, escribió un libro sobre la liberalización, que se llama Privatization, y cita la privatización de la recolección en Buenos Aires como uno de los mejores ejemplos en el mundo". Tanto Laura como Della Paolera destacan que el proceso de adjudicación de la licitación de los residuos se jugaba sobre un criterio de excelencia técnica, en que los norteamericanos se destacaban respecto de las ocho empresas argentinas, descartadas por "incapacidad técnica. Sin saber el precio ni nada, sino solo sobre antecedentes" (Laura, entrevista personal, 24 de junio de 2010). Aunque en los papeles la ciudad de Buenos Aires fuera dividida en dos áreas de adjudicación, una controlada por la CEAMSE y otra por la Ciudad de Buenos Aires, el conjunto fue adjudicado a la misma empresa, Waste Management Inc., que se asoció con el grupo argentino SOCMA (perteneciente a la familia Macri) para crear la firma Manliba. La privatización fue importante entonces en la medida en que la adjudicación única del servicio estandarizó bajo criterios únicos la infraestructura, el material, la frecuencia, el personal, cuando en los años previos a ella los esfuerzos de racionalización del servicio fueron entorpecidos por la fuerte fragmentación de los equipos de operación municipal, y por el carácter vetusto y disparejo de los camiones. En el discurso de los actores existe un fuerte contraste entre este proceso de privatización en la Ciudad y el que ocurrió en los municipios del conurbano, que pone de relieve una vez más la vocación representativa del espacio céntrico respecto del conurbano, un lugar menos conocido por los ejecutivos de la CEAMSE.

Finalmente, la transformación institucional de la gestión de los residuos remite la discusión al carácter metropolitano del dispositivo tecnológico, volviendo al principio del análisis: ¿cómo el cinturón ecológico se instaura como marca metropolitana? Numerosos artículos de diarios, conferencias y ponencias insisten sobre el carácter transjurisdiccional de las competencias de la empresa de Estado. El CEAMSE se creó el 3 de enero de 1977, por el Decreto Ley 9111/77, mediante 
un convenio firmado por la provincia de Buenos Aires y la Ciudad de Buenos Aires, bajo la supervisión del poder nacional. Esta creación institucional constituye una verdadera renovación del aparato político-institucional: supera las divisiones político-administrativas en los partidos del conurbano bonaerense y facilita la transferencia de presupuesto público a empresas privadas contratistas. La implementación del organismo se hizo posible por la retirada de la vida política a un nivel muy local (González Bombal, 1988), debido a la presencia del régimen de facto. La implementación de la CEAMSE representó, para los municipios, una medida autoritaria que los obligaba a destinar un porcentaje muy alto de su presupuesto, que para algunos llegó a alcanzar el 40\%, a la recolección y a la disposición final de sus residuos (González Bombal, 1988). Este esfuerzo se debe, entre otras cosas, a la necesidad de privatización de la recolección que tienen los municipios. Pero se debe también a la fijación de tarifas internacionales para la disposición de los residuos. Sin embargo, esta cuestión no está tratada como tal en los diarios o en los trabajos sobre el cinturón ecológico y no aparece sino implícitamente en las notas sobre el aumento de los impuestos municipales. Ahí emerge el margen de maniobra entre la promoción que se hace del cinturón ecológico y el orden cronológico de las operaciones: mientras en los diarios es la traza verde la que se promociona, la que aparecerá a mediano plazo, las obras implementadas a corto plazo son los rellenos sanitarios. Al concentrar los residuos del conurbano bonaerense se activa también una concentración de capital proveniente de la facturación directa del CEAMSE a los municipios. En los hechos, el CEAMSE funciona como una "bomba colectora" de capitales que se redistribuye hacia las empresas contratistas que operan los rellenos sanitarios.

\section{Conclusiones: ¿̨la inversión de la imagen?}

En este texto queríamos demostrar por qué el cinturón ecológico se instaló en la memoria urbana colectiva de Buenos Aires y hasta superó su realización física. Abordamos, bajo distintos ángulos, la cuestión de la promoción urbana del emprendimiento, para cuestionar sucesivamente su lugar en el marco de una crisis urbana y la difusión de su trazado en la metrópoli como proyecto de ciudad. Además, pusimos en tela de juicio el carácter innovador de la tecnología de los rellenos sanitarios y de la fórmula institucional propuesta con la creación de una empresa de Estado. El análisis del conjunto de estas operaciones puso de relieve que la cuestión ecológica, abordada esencialmente desde el punto de vista de la calidad de vida y de la preservación de la salud, así como de los recursos naturales, fue uno de los temas de promoción urbana más importantes del Buenos Aires del gobierno cívicomilitar de 1976 a 1983. Por este medio, los equipos gubernamentales apuntaban a mostrar una ciudad que podía insertarse en los debates de su tiempo e igualar los modelos en que se inspiraba: las ciudades norteamericanas y europeas.

Si bien el carácter inacabado del proyecto no permite distinguirlo de otras numerosas experiencias de planificación llevadas a cabo en la Argentina, hoy en día es más bien la dimensión autoritaria la que resurge en las protestas contra los rellenos 
sanitarios. Grupos de vecinos reclaman el derecho a un ambiente sano en un contexto más general, mientras la reivindicación de asociaciones y organizaciones no gubernamentales por el ambiente se ha dirigido hacia una mayor judicialización (Merlinsky, 2009), lo que significa que estos grupos enmarcan su demanda dentro de cuadros legales que, en teoría, tendrían que protegerlos contra los efectos nefastos de instalaciones como los rellenos sanitarios. En el caso de Buenos Aires, los eslóganes "CEAMSE MATA" agrupan bajo una misma acusación a los sitios de disposición, el proyecto original y el contexto autoritario de su creación. El proyecto pasó de encarnar una infraestructura de ordenamiento territorial asociada a las mejores tecnologías de los años setenta, a cristalizar las aporías de un sistema basado en la acumulación indiferenciada y cuantitativa de los residuos. Aunque las estrategias desplegadas por la CEAMSE apunten a reconvertir tanto las actividades como la imagen del organismo para adecuarlas a los nuevos parámetros de la gestión sustentable de los residuos, el ente metropolitano queda asociado con las dificultades político-administrativas, las apuestas económicas y las dificultades organizativas que rodean la implementación de una "gestión integral de los residuos sólidos urbanos". Sin embargo, esta promoción "al revés" no pone un punto final a la discusión sobre la simbología del emprendimiento. Como lo indican Azuela y Mussetta (2008) para los parques nacionales en México, todo conflicto ambiental tiene "algo más que el ambiente", justamente. La cuestión ambiental sirvió para promover a Buenos Aires como una de las capitales latinoamericanas más vanguardistas y exclusivas de su tiempo en los años setenta. Hoy en día, a la par de la emergencia de la problemática ambiental y el cierre de los rellenos sanitarios, aparecen demandas de acceso a los servicios urbanos en conjunto con reivindicaciones de mayor alcance, como es el derecho a la ciudad. OEURE

\section{Referencias bibliográficas}

Alegre, M. (1978). Consideraciones sobre las conclusiones del simposio regional sobre desechos sólidos. Ponencia presentada en el marco del Quinto Congreso Argentino de Saneamiento organizado por la Administración General de Obras Sanitarias de la Nación. Santa Fe, Argentina.

Azuela, A. \& Mussetta, P. (2008). "Quelque chose de plus" que l'environnement. Conflits sociaux dans trois aires naturelles protégées du Mexique. Dossier Amérique latine, conflits, environnement. Problèmes d'Amérique Latine, 70, 13-39. En http://www.cairn.info/revue-problemes-d-amerique-latine-2008-4-page-13.htm / doi: 10.3917/pal.070.0013.

Bellucci, A., Gil, J. \& García, J. (1982). Recuperación de áreas deterioradas. En Primer Congreso de Ecología Urbana. Buenos Aires, Argentina.

Bielsa Lila, O. B., Abramovitch, B. \& Virgil, R. (1978). Influencia de los rellenos sanitarios en la calidad del agua subterránea. En Tercer congreso Argentino de Saneamiento. Santa Fe, Argentina.

Coordinación Ecológica Área Metropolitana Sociedad del Estado (CEAMSE). (1978). Memorias y Balances. Buenos Aires: Ediciones CEAMSE.

Coordinación Ecológica Área Metropolitana Sociedad del Estado (CEAMSE). (1974). Cuarto seminario técnico sobre la contaminación urbana. Organizado por la Fundación Gillette. Buenos Aires, Argentina. 
David, P. A. (1985). Clio and the Economics of QWERTY. American Economic Review, 75(2): 332 337. En http://www.econ.ucsb.edu/ tedb/Courses/Ec100C/DavidQwerty.pdf

Del Cioppo, G. (1980). Declaraciones del titular de la CMV [Comisión Municipal de Vivienda] Guillermo del Cioppo. Competencia, 191 (marzo). En http://www.comisionporlamemoria. org/jovenesymemoria/docs/fichas/erradicacion-villas.pdf

Dubois-Maury, J. (1990). Les villes argentines: une urbanisation sans urbanisme? Annales de Géographie, 99(556), 695-714. En http://www.persee.fr/web/revues/home/prescript/article/ geo_0003-4010_1990_num_99_556_21005

Dumont, M. \& Devisme, L. (2006). Les métamorphoses du marketing urbain. Espacetemps. En http:// test.espacestemps.net/articles/les-metamorphoses-du-marketing-urbain/

González Bombal, M. I. (1988). Los vecinazos. Las protestas barriales en el Gran Buenos Aires 19821983. Buenos Aires: Ediciones del IDES.

Gorelik, A. (1998). La grilla y el parque. Espacio público y cultura urbana en Buenos Aires, 1887-1936. Bernal: Universidad Nacional de Quilmes.

Greenberg, M. (2008). Branding New York: How a city in crisis was sold to the world. London: Routledge.

Laura, G. (1979). El cinturón ecológico (2a ed. rev. y aum.). Buenos Aires: Ediciones CEAMSE (Coordinación Ecológica Área Metropolitana Sociedad del Estado).

Liernur, F. (1993). La ciudad efímera. En G. Silvestri G. \& F. Liernur, El umbral de la metrópolis (pp. 177-222). Buenos Aires: Sudamericana.

Melosi, M. (2004). Garbage in the cities. Refuse, reform and the environment (Ed. rev.). Pittsburgh, PA: University of Pittsburgh Press.

Merlinsky, G. (2009). Atravesando el río: la construcción social y politica de la cuestión ambiental en Argentina. Dos estudios de caso entorno al conflicto por las plantas de celulosa en el río Uruguay $y$ al conflicto por el saneamiento de la cuenca del Matanza-Riachuelo. Tesis de doctorado en Ciencias Sociales y en Geografía, Universidad de Buenos Aires (UBA) - Paris 8.

Municipalidad de la Ciudad de Buenos Aires (1976). Autopista 25 de Mayo - Autopista Perito Moreno. Buenos Aires: Municipalidad de la Ciudad de Buenos Aires.

Oszlak, O. (1991). Merecer la ciudad: los pobres y el derecho al espacio urbano. Buenos Aires: Centro de Estudios de Estado y Sociedad (CEDES)-Humanitas.

Paiva, V. (1999). Medio ambiente urbano: Una mirada desde la historia de las ideas científicas y las profesiones de la ciudad. Buenos Aires 1850-1915. Ponencia presentada en el marco de la Conferencia Internacional La Cultura Arquitectónica hacia 1900. Revalorización crítica y preservación patrimonial. Buenos Aires, Argentina. En http://revistaurbanismo.uchile.cl/n3/paiva.html

Paiva, V. (2003). De los huecos a los rellenos sanitarios. Revista Cientifica UCES, 10(1), 112-134.

Peyrrefitte, A., Schmelck, R. \& Dumoulin, R. (1977). Réponse à la violence: rapport à M. le Président de la République. Paris: La Documentation Française. En http://www.ladocumentationfrancaise.fr/rapports-publics/774023100/index.shtml

Prignano, A. (1998). Crónica de la basura porteña. Del fogón indígena al cinturón ecológico. Buenos Aires: Junta de Estudios Históricos de San José de Flores.

Randle, P. (Comp.). (1977). Buenos Aires y sus problemas urbanos. Trabajos originales de Carlos María Della Paolera. Buenos Aires: Oikos.

Ríspoli, J., Leva, A., Crivelari, C. \& Comerci, J. (1962). Contaminación aérea producida por la incineración y destrucción de los residuos de la Ciudad de Buenos Aires. Ponencia presentada 
en el marco de la Primera Conferencia Latinoamericana de Contaminación del Aire. Buenos Aires, Argentina.

Rosemberg-Lasorne, M. (1997). Marketing urbain et projet de ville: parole et représentations géographiques des acteurs. Cybergeo: European Journal of Geography. En http://cybergeo.revues. org/ 1977

Schvarzer, J. (1981). Expansión económica del estado subsidiario. Buenos Aires: Centro de Investigaciones Sociales sobre el Estado y la Administración (CISEA).

Suárez, F. (1972). Tercer seminario técnico sobre la contaminación urbana. Organizado por la Fundación Gillette. Buenos Aires, Argentina.

Suárez, F. (1998). Que las recojan y arrojen fuera de la ciudad. Colección Documento de Trabajo, 8. San Miguel: Publicaciones de la Universidad Nacional de General Sarmiento (UNGS).

\section{Extractos de diarios nacionales}

(1976, 23 de abril). Dramático cuadro de la situación de la Comuna trazó en su mensaje el intendente Cacciatore. Clarín, p. 12.

(1976, 31 de diciembre). Será combatida en la ciudad la contaminación ambiental. Clarín, p. 19.

(1977, 8 de enero). Parques recreativos. Plan para un sistema regional. Clarín, p. 12.

(1977, 14 de enero). La muerte del incinerador. Clarín, p. 12.

(1977, 21 de abril). Un cinturón verde para Buenos Aires. Clarín, p. 21.

(1977, 6 de mayo). La comuna porteña y el gobierno bonaerense se comprometen en una obra de desarrollo regional. Con veinte años de demora. Clarín, p. 14.

(1977, 7 de mayo). Plan para la recuperación ecológica. Clarín, p. 16.

(1977, 13 de mayo). El necesario planeamiento del futuro urbano de Buenos Aires. Clarín, p. 14.

(1977, 13 de mayo). Inventaron en Alemania petróleo artificial a partir de la basura. Clarín, p. 13.

(1978, 14 de mayo). "Buenos Aires for export”. Clarín, p. 24.

(1977, 4 de junio). Editorial, Cinturón ecológico. Clarín, p. 6.

(1977, 9 de noviembre). Qué es el urbanismo. Clarín, p. 17.

(1978, 20 de enero). Contrataron la construcción de dos autopistas. Clarín, p. 12.

(1978, 12 de abril). Los beneficios del relleno sanitario. Clarín, p. 20.

(1978, 14 de abril). ¿Qué le reclaman los porteños a su intendente? Clarín, pp. 16-17.

(1978, 6 de junio). Los ingenieros y la Argentina. Clarín, p. 14.

(1976, 26 de septiembre). La batalla por los espacios verdes. La Nación, pp. 12-16.

(1977, 7 de mayo). Convenio sobre espacios verdes. La Nación, p. 20.

(1977, 19 de junio). Nueva era - combustible de basura. La Nación, p. 26.

(1977, 25 de agosto). Algunos problemas que ocasiona el progreso. La Nación, p. 14. 